\title{
Spectral Evolution Models for the Next Decade
}

\author{
Claus Leitherer \\ Space Telescope Science Institute, 3700 San Martin Dr., Baltimore, MD 21218, USA \\ email: leitherer@stsci.edu
}

\begin{abstract}
Spectral evolution models are a widely used tool for determining the stellar content of galaxies. I provide a review of the latest developments in stellar atmosphere and evolution models, with an emphasis on massive stars. In contrast to the situation for low- and intermediatemass stars, the current main challenge for spectral synthesis models are the uncertainties and rapid revision of current stellar evolution models. Spectral libraries, in particular those drawn from theoretical model atmospheres for hot stars, are relatively mature and can complement empirical templates for larger parameter space coverage. I introduce a new ultraviolet spectral library based on theoretical radiation-hydrodynamic atmospheres for hot massive stars. Application of this library to star-forming galaxies at high redshift, i.e., Lyman-break galaxies, will provide new insights into the abundances, initial mass function and ages of stars in the very early universe.
\end{abstract}

Keywords. line: profiles - stars: early-type - stars: evolution - stars: mass loss — galaxies: high redshift — galaxies: stellar content — ultraviolet: stars

\section{Introduction}

Spectral evolution models attempt to reproduce the observed spectra of systems ranging in sizes from star clusters to luminous galaxies by numerically combining models or observations of star formation, stellar evolution, and stellar spectra. Tinsley (1968) is generally credited for initiating the field, and Charlot \& Bruzual's (1991) introduction of the isochrone synthesis technique validated this approach for ages up to a Hubble time. The success of synthetic spectral models echoes the increased availability of computers in astronomy. The power of this technique has been amplified over the past decade by the growing importance of the internet and databases for storage, query, and distribution of spectral templates and related models. This trend is revolutionizing the field in a way similar to Tinsley's and Charlot's \& Bruzual's achievements.

In this talk I will concentrate less on the technical but more on some of the astrophysical aspects of spectral evolution models. After a brief discussion of the main ingredients in the model I will address the two major components: stellar evolution models and stellar libraries. The emphasis will be on massive stars, for which stellar evolution still is the major challenge, whereas reliable stellar libraries are becoming available in larger and larger numbers. This situation is somewhat opposite to the case of low-mass stars. I will introduce a new ultraviolet (UV) spectral library constructed from a grid of radiation-hydrodynamic models and discuss its potential for interpreting the rest-frame UV spectra of Lyman-break galaxies. Detecting the first stellar generations and modeling and understanding their spectra will be a major effort for the next decade. 


\section{Main Physics Input}

Evolutionary spectral synthesis is a special case of spectral synthesis whose goal is to reproduce observed spectra self-consistently from the star-formation history of a galaxy and from stellar evolution models. The advantage of this method compared with spectral synthesis are the smaller number of free parameters and the increased predictive power. The obvious price to pay is the reliance on models and idealized empirical parameters whose failure may doom the calculated synthetic spectra (Cid Fernandes et al. 2005). Since I am focusing on evolutionary synthesis in the following, assessing these assumptions becomes critical.

The astrophysical ingredients entering evolutionary spectral synthesis fall into four categories: (i) Quantities related to the star-formation process, i.e., the star-formation rate and its evolution with time and the stellar mass spectrum at birth, also known as the initial mass function (IMF). Both are usually considered free parameters and will not be discussed here. See, e.g., the conference proceedings of Corbelli et al. (2005) and the review by Kroupa (2007). (ii) Once stars have formed, a prescription for the evolution of luminosity $(L)$, effective temperature $\left(T_{\text {eff }}\right)$, and mass $(M)$ as a function of time and initial mass and chemical composition is needed. This prescription is provided by stellar evolution models. (iii) Spectral libraries describe the emergent spectrum of each star for any given $\left(L, T_{\text {eff }}, M\right)$. These libraries can be either empirical or theoretical. (iv) Secondorder effects, such as dust reddening or geometric effects are often accounted in a very approximate way or even neglected altogether. Reddening by dust is of course a major issue when dealing with the UV. See the review by Calzetti (2001).

The areas of massive star evolution and stellar libraries are particularly active, with major revisions and anticipated new breakthroughs. I will therefore concentrate on these two subjects in what follows.

\section{Stellar Evolution}

Until very recently the evolution of massive stars was thought to be determined by the chemical composition, stellar mass, and mass-loss rate, plus atomic physics and some second-order effects. The evolution models suggested reasonable agreement with observations of both individual stars and stellar populations. Subsequently the key role of stellar rotation in the evolution of massive stars was recognized (Meynet 2009). Evidence of anomalous stellar surface abundances already on the main-sequence, the lifetimes of certain evolutionary phases, and revised lower mass-loss rates support the concept of rotation.

Rotation modifies the hydrostatic structure, induces additional mixing and affects the stellar mass loss. Generally, rotation increases both $L$ and $T_{\text {eff }}$ in massive stars. This is the result of the larger convective core and the lower surface opacity for higher rotation speed. (Recall that hydrogen is the major opacity source and any decrease of its relative abundance by mixing lowers the opacity and therefore increases the temperature.) The most dramatic changes with respect to models without rotation occur at the short-wavelength end of the spectrum. The ionizing luminosities for a single stellar population with mass $10^{6} M_{\odot}$ are shown in Fig. 1 . Since the most massive stars are more luminous and hotter, their ionizing luminosities increase during O-star dominated phases $(2-10 \mathrm{Myr})$. The increase reaches a factor of 3 in the hydrogen ionizing continuum and several orders of magnitude in the neutral and ionized helium continua. The predictions for the latter need careful scrutiny, as the photon escape fraction crucially depends on the interplay between the stellar parameters supplied by the evolution models and the 


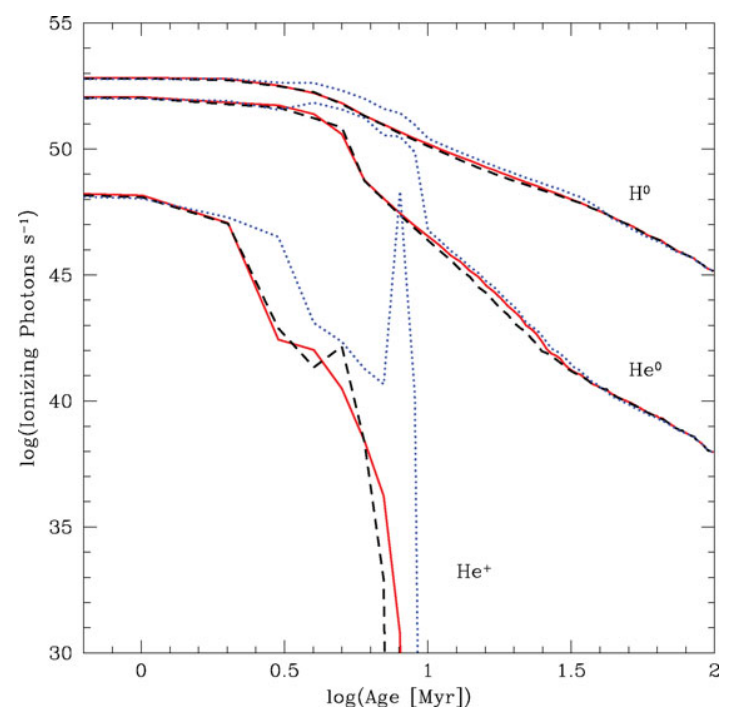

Figure 1. Number of photons in the $\mathrm{H}^{0}, \mathrm{He}^{0}$, and $\mathrm{He}^{+}$continuum (solar composition). Models without $v_{\text {rot }}=300 \mathrm{~km} \mathrm{~s}^{-1}$ (solid) and with $v_{\text {rot }}=0$ (dotted). The dotted curve denotes an alternative exploratory model with $v_{\text {rot }}=0$. See Vázquez et al. (2007) for details.

radiation-hydrodynamics of the atmospheres. In contrast, the escape of the hydrogen ionizing photons has little dependence on the particulars of the atmospheres and consequently is a relatively safe prediction - if the stellar evolution models themselves can be trusted.

Stellar winds from hot stars are a second area in which major changes have occurred. If our basic understanding of the evolutionary connections between different stellar species is correct, strong mass loss must occur in order to decrease the mass of an initially massive O star to that of a much less massive Wolf-Rayet star or red supergiant. Until recently, observational mass-loss rates were thought to be sufficiently well understood that they could serve as a fixed input for evolution models (Maeder \& Conti 1994). The discovery of wind inhomogeneities with the result of much lower mass-loss rates (Fullerton et al. 2006; Puls et al. 2008) and of eruptive phases (Smith 2010) have dramatically changed this perspective. The uncertainties of the observed rates can easily reach factors of several. Therefore mass loss in evolution models is now considered an adjustable parameter that together with rotation governs the stellar properties with time.

The downward revision of the mass-loss rates of unevolved and evolved luminous, massive stars poses a severe challenge for the standard stellar evolution paradigm. Consider a $40 M_{\odot} \mathrm{O}$ star on the main-sequence, which is thought to become a Wolf-Rayet star on a time-scale of $\sim 5$ Myr. Assuming a typical Wolf-Rayet mass of $15 M_{\odot}$, the average mass-loss rate prior to entering the Wolf-Rayet phase should be $\sim 10^{-5} M_{\odot} \mathrm{yr}^{-1}$, almost an order of magnitude higher than the observed O-star rates. Smith (2010) emphasized the potential role of eruptive phases of massive stars to compensate for the lower steadystate rates. During a short evolutionary phase massive stars could lose as much mass as during the entire O-star phase with a steady wind.

Both the effects of rotation and the uncertain mass-loss rates make stellar evolution models of massive stars much less certain than previously thought, in particular for postmain-sequence evolution. Therefore spectral evolution models relying on such phases must be viewed with care. 


\section{Spectral Libraries}

Spectral libraries can be built from empirical or theoretical stellar spectra. The pros and cons of either approach are discussed extensively in the literature (e.g., Gustaffson et al. 2007; Martins \& Coelho 2007).

Arguments in favor of empirical libraries are:

- Laboratory data are often lacking and therefore the quality and completeness of the line lists used for the computation of stellar atmospheres are insufficient.

- The computational effort for generating a large grid of models can be formidable. Even if individual models matching the observations can be produced, they are not suitable for inclusion in a library if the required parameter space cannot be covered.

- Departures from local thermal equilibrium (LTE) can be significant and greatly complicate the computational effort.

- The most luminous stars have stellar winds and are extended. Therefore model atmospheres must account for sphericity effects and often include hydrodynamics.

- Deviations from spherical symmetry can be important, as well as depth effects in the photosphere. As a result, plane-parallel, one-dimensional models are no longer valid.

On the other hand, limitations of empirical libraries are:

- Massive stars are rare. Assembling a complete library, in particular at non-solar chemical composition, can be a severe challenge.

- Telescope time is expensive, whereas computer time is cheap. Using a telescope for building a large stellar library is often impractical.

- Interstellar reddening by dust for massive stars is not negligible. This is particular true in the UV, where large reddening corrections must be applied to observations.

- Related to the previous item are interstellar absorption lines in the UV. Such lines are blended with stellar lines and are almost impossible to correct for.

- An often overlooked issue is the need of a spectral-type vs. $T_{\text {eff }}$ relation when empirical libraries are linked to evolution models. This relation is derived from models, so that ultimately empirical libraries depend on the same atmosphere models they are intended to replace.

When assessing the relevance of the above points for different classes of massive stars, it turns out that concerns about theoretical stellar spectra mostly apply to cool stars. The low temperatures of late-type stars magnify the challenges for atmosphere modeling so that cool, massive stars should be represented by empirical spectra in spectral evolution models. The opposite can be said for hot stars. Here, the atmosphere models are rather mature (Puls 2008) and any remaining shortcomings by far outweigh the drawbacks of observational libraries, such as parameter space coverage and contamination by interstellar gas and dust.

Following this philosophy, we have been pushing for a fully theoretical UV stellar library for implementation in the Starburst99 synthesis code (Leitherer et al. 1999; Vázquez \& Leitherer 2005; Leitherer \& Chen 2009). The main science driver are the restframe UV spectra of Lyman-break galaxies whose spectral features may hold the clue for understanding star formation at redshifts of 3 and higher. Lyman-break galaxies are massive $\left(M \approx 10^{11} M_{\odot}\right)$, mildly metal-poor $(\mathrm{O} / \mathrm{H}+12 \approx 8.2)$, UV-bright $(E(B-V) \approx 0.1)$ galaxies currently forming stars at rates of order $10^{2} M_{\odot} \mathrm{yr}^{-1}$ (Giavalisco 2002). Prior attempts to model their rest-frame UV spectra using an empirical library were quite successful (e.g., Quider et al. 2009) but the limitations are obvious: the lack of spectra of metal-poor massive stars, the presence of $\alpha$-element/Fe variations, and the generally 

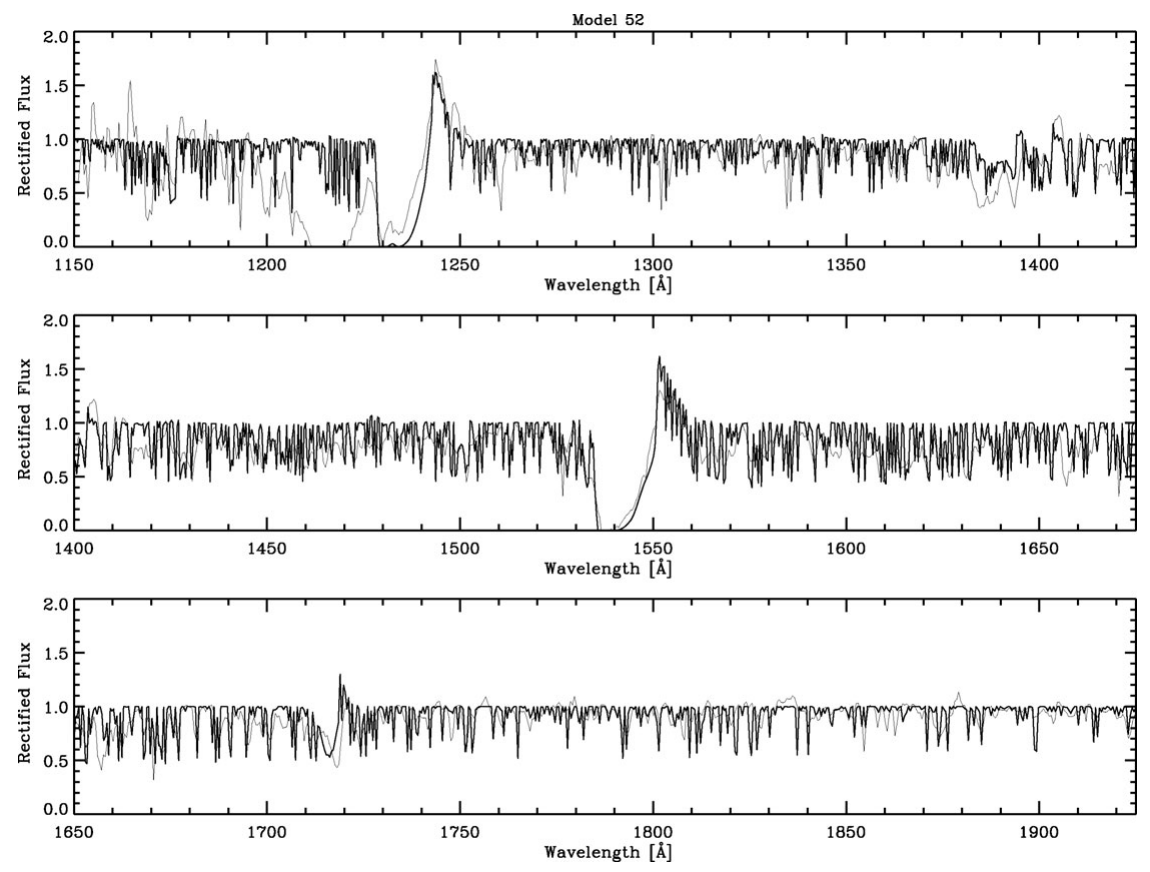

Figure 2. Comparison of a theoretical stellar UV spectrum with parameters $\log L=5.3, T_{\text {eff }}=$ $34,500 \mathrm{~K}, \log g=3.7$ (thick line) and an average IUE spectrum of three O7.5 III stars (thin). The observed feature at $1216 \AA$ is interstellar Lyman- $\alpha$. From Leitherer et al. (2010).

low $\mathrm{S} / \mathrm{N}$ of the UV spectra of the template stars. A first effort to model Lyman-break rest-frame UV spectra purely theoretically was done by Rix et al. (2004), who matched the weak photospheric features in the spectra to determine stellar chemical abundances. The state of the stellar atmospheres at that time precluded the modeling of the stellarwind lines in the spectra, which is frustrating because the wind lines are the strongest spectral features and can be detected even in low-S/N spectra of Lyman-break galaxies.

Substantial astrophysical (e.g., inclusion of wind structure and X-ray ionization) and technical (e.g., decrease of computing time by an order of magnitude) improvements in atmospheric modeling are now allowing us to compute the full spectrum, including the wind lines (Leitherer et al. 2010). We used WM-Basic, a non-LTE, spherically extended, blanketed, radiation-hydrodynamics code for hot stars (Pauldrach et al. 2001) for a selfconsistent calculation of the photospheric and wind parameters and the generation of the synthetic spectrum between 900 and $3000 \AA$. The library covers the relevant parameter space of hot stars with $M \geqslant 5 M_{\odot}$ on and off the main-sequence having abundances between twice and $10^{-2}$ solar. The spectral resolution is $0.5 \AA$. An example is shown in Fig. 2 where one particular model is compared to the average observed IUE spectrum of three stars with closely matching spectral types. The most prominent lines are $\mathrm{N} \mathrm{V}$ $1240 \AA$, Si IV $1400 \AA$, C IV $1550 \AA$, and N IV $1720 \AA$. Keeping in mind that the slight differences between some features are entirely attributable to mismatches of stellar parameters, the agreement is excellent and gives confidence in this method.

We then implemented the stellar library in Starburst99 where it complements and extends the empirical libraries of Robert et al. (1993), de Mello et al. (2000), Leitherer et al. (2001), and Pellerin et al. (2002) currently in use. As a first test we compared the synthetic UV spectra for a standard stellar population computed with both methods. This comparison is shown in Fig. 3. There are no significant differences that would indicate 

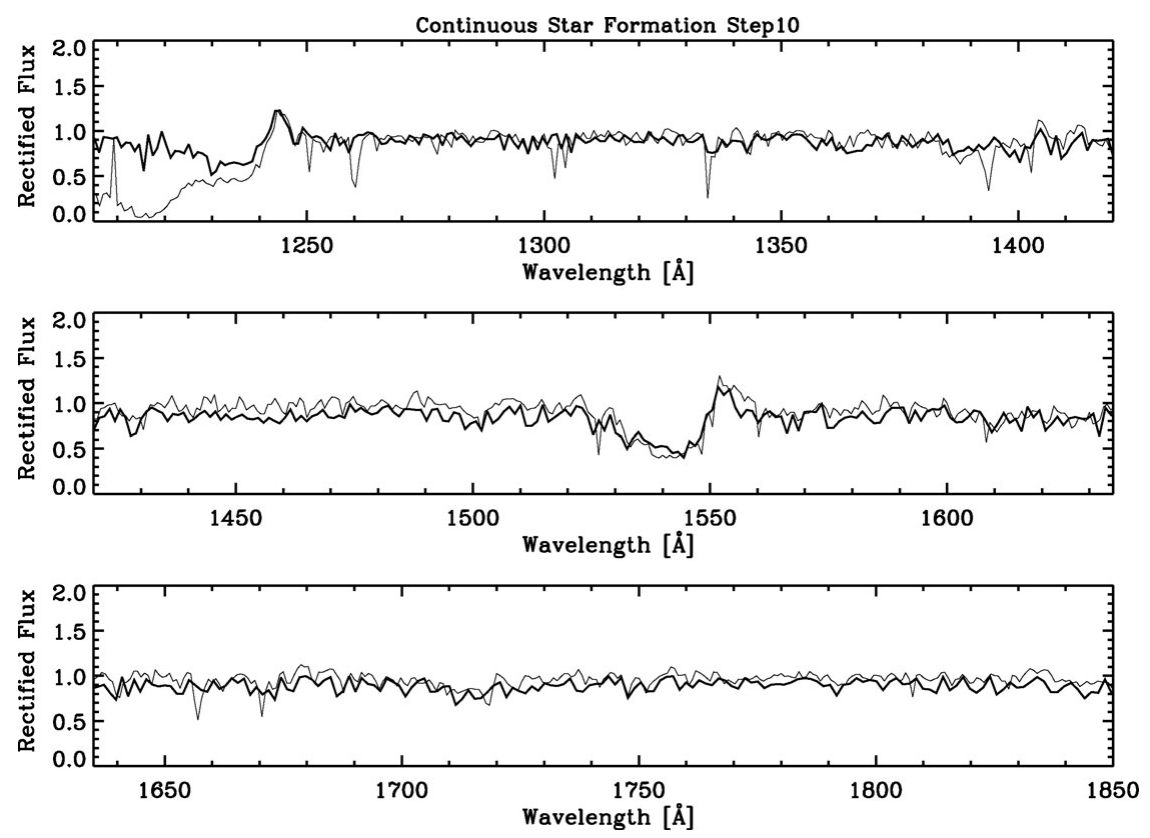

Figure 3. UV spectrum of a standard stellar population with theoretical (thick line) and empirical (thin) libraries in Starburst99. The spectral resolution of the theoretical spectra has been degraded to $1.0 \AA$ to match the empirical spectra. Solar metallicity; Salpeter IMF; age $10 \mathrm{Myr}$; continuous star formation. From Leitherer et al. (2010).

deficiencies in the models. Several issues are worth noting. (i) The continuum level in the theoretical spectrum is known a priori and no manual normalization is necessary, whereas such a step had to be performed for the empirical library. The higher continuum level of the empirical spectrum indicates a systematic error introduced by the normalization. (ii) N V $1240 \AA$ in the empirical spectrum is contaminated by Galactic Lyman- $\alpha$, whereas this effect is not an issue in the theoretical spectrum. (iii) The empirical spectrum is contaminated by narrow interstellar absorption lines which arise along the sight lines to the individual template stars. Examples are C II $1335 \AA$ and Si II $1260 \AA$ A. Even more disturbing is the contamination of the Si IV $1400 \AA$ stellar-wind line. The theoretical library allows an analysis without these biases.

A key application of the newly developed theoretical library will be the determination of stellar abundances in Lyman-break galaxies. Photospheric and stellar-wind lines respond to abundance variations in a different manner. Whereas photospheric lines are sensitive to excitation/ionization and abundance effects, stellar-wind lines are in addition predominantly controlled by the stellar mass-loss rate and velocity field. Since the massloss rate and velocity field themselves are a function of chemical composition, the strong UV lines, such as N V $1240 \AA$, Si IV $1400 \AA$, or C IV $1550 \AA$ become quite sensitive to abundance variations despite the fact that they are deeply saturated. This point is illustrated in Fig. 4, which shows the variation of N V, Si IV, and C IV with metallicity. All three lines display more or less significant changes with metallicity, making them suitable as abundance diagnostics. The N V line, however, is essentially metallicity independent until $Z=0.05 Z_{\odot}$ is reached. The behavior of the $\mathrm{N} \mathrm{V}$ line results from the counteracting effects of chemical composition and stellar temperature on the $\mathrm{N}^{4+}$ column density. The dominant ionization stage in $\mathrm{O}$ star winds is $\mathrm{N}^{3+}$, and the mean ionization fraction of $\mathrm{N}^{4+}$ increases monotonically with stellar temperature. Therefore, to first 

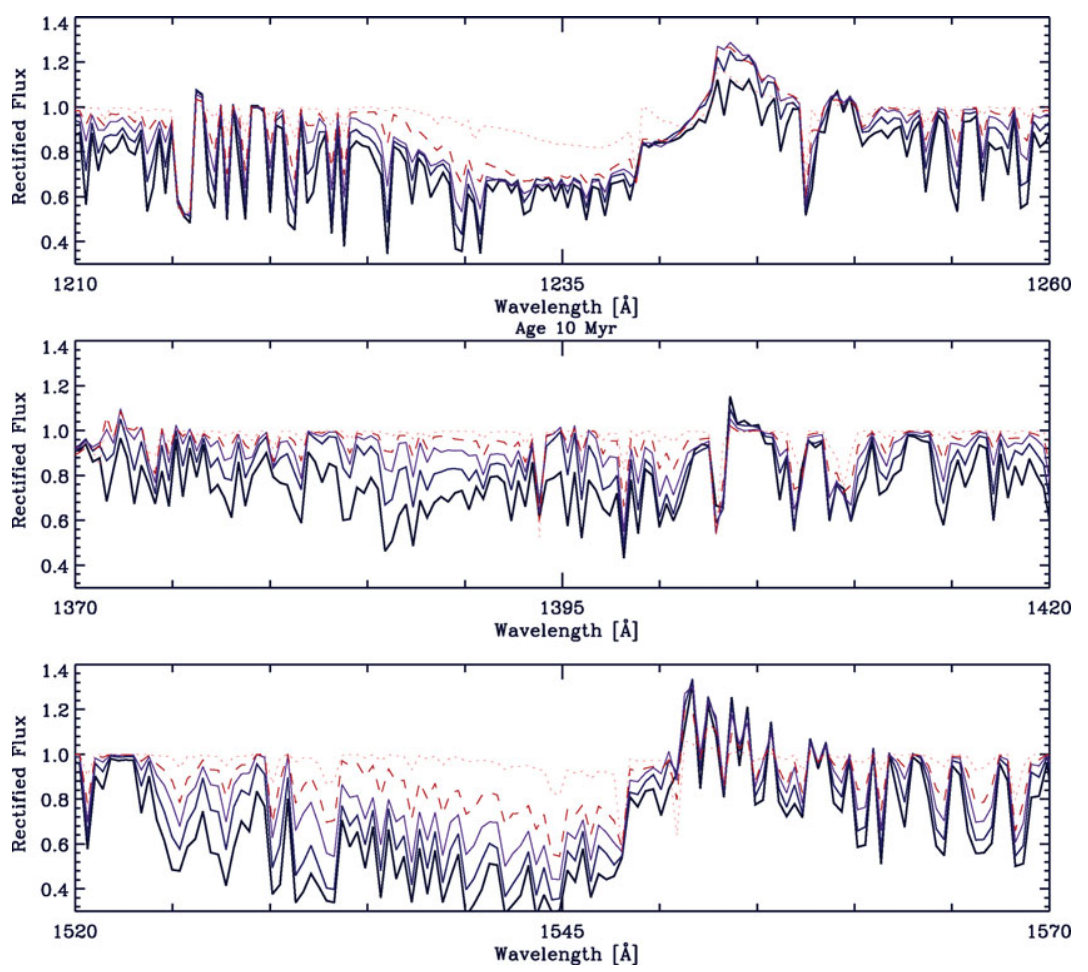

Figure 4. Variation of N V $1240 \AA$ (top), Si IV $1400 \AA$ (middle), or C IV $1550 \AA$ (bottom) with metallicity. The five metallicities plotted are $2 Z_{\odot}$ (thick solid), $Z_{\odot}$ (solid), $0.4 Z_{\odot}$ (thin solid), $0.2 Z_{\odot}$ (dashed), and $0.05 Z_{\odot}$ (dotted). The population parameters are as in Fig. 3. From Leitherer et al. (2010).

order, the $\mathrm{N}^{4+}$ column density becomes independent of the stellar mass loss because the ionization fraction and the total nitrogen column density have the opposite dependence on the mass-loss rate. This effect was discussed before by Leitherer et al. (2001).

The different functional behavior of the various stellar-wind lines allows the discrimination between the often degenerate effects of abundance, age, and IMF. This is of particular interest at higher and higher redshift and earlier stellar generations, which are expected to have an IMF biased towards very massive stars.

\section{Acknowledgements}

Support for this work was provided by NASA Grant N1317 and by the STScI Director's Discretionary Research Fund. Part of this research was done by Paula Ortiz (University of Medellín) as her project during the 2009 STScI Space Astronomy Summer Program.

\section{References}

Calzetti, D. 2001, PASP, 113, 1449

Charlot, S. \& Bruzual, G. 1991, ApJ, 367, 126

Cid Fernandes, R., Mateus, A., Sodré, L., Stasińska, G., \& Gomes, J. M. 2005, MNRAS, 358, 363

Corbelli, E., Palla, F., \& Zinnecker, H. 2005, The Initial Mass Function 50 Years Later (Doordrecht: Springer)

de Mello, D. F., Leitherer, C., \& Heckman, T. M. 2000, ApJ, 530, 251

Fullerton, A. W., Massa, D. L., \& Prinja, R. K. 2006, ApJ, 637, 1025 
Giavalisco, M. 2002, ARA\&A, 40, 579

Gustafsson, B., Heiter, U., \& Edvardsson, B. 2007, in: A. Vazdekis \& R. F. Peletier, IAU Symp. 241, Stellar Populations as Building Blocks of Galaxies (Cambridge: CUP), p. 47

Kroupa, P. 2007, in: A. Vazdekis \& R. F. Peletier, IAU Symp. 241, Stellar Populations as Building Blocks of Galaxies (Cambridge: CUP), p. 109

Leitherer, C. \& Chen, J. 2009, New Astronomy, 14, 356

Leitherer, C., Leão, J. R. S., Heckman, T. M., Lennon, D. J., Pettini, M., \& Robert, C. 2001, ApJ, 550, 724

Leitherer, C. et al. 1999, ApJS, 123, 3

Leitherer, C., Ortiz, P. A., Bresolin, F., Kudritzki, R.-P., Lo Faro, B., Pauldrach, A. W. A., \& Pettini, M. 2010, ApJS, in preparation

Maeder, A. \& Conti, P. S. 1994, ARA $\& A$ A, 32, 227

Martins, L. P. \& Coelho, P. 2007, MNRAS, 381, 1329

Meynet, G. 2009, Lecture Notes in Physics (Berlin: Springer), 765, 139

Pauldrach, A. W. A., Hoffmann, T. L., \& Lennon, M. 2001, A\& A, 375, 161

Pellerin, A. et al. 2002, ApJS, 143, 159

Puls, J. 2008, in: F. Bresolin, P. A. Crowther, \& J. Puls, IAU Symp. 250, Massive Stars as Cosmic Engines (Cambridge: CUP), p. 25

Puls, J., Vink, J. S., \& Najarro, F. 2008, A\&A Rev., 16, 209

Quider, A. M., Pettini, M., Shapley, A. E., \& Steidel, C. C. 2009, MNRAS, 398, 1263

Rix, S. A., Pettini, M., Leitherer, C., Bresolin, F., Kudritzki, R.-P., \& Steidel, C. C. 2004, ApJ, 615,98

Robert, C., Leitherer, C., \& Heckman, T. M. 1993, ApJ, 418, 749

Smith, N. 2010, in: C. Leitherer et al., Hot and Cool: Bridging Gaps in Massive Star Evolution (San Francisco: ASP), in press

Tinsley, B. M. 1968, ApJ, 151, 547

Vázquez, G. A. \& Leitherer, C. 2005, ApJ, 621, 695

Vázquez, G. A., Leitherer, C., Schaerer, D., Meynet, G., \& Maeder, A. 2007, ApJ, 663, 995 